\title{
Power communication network traffic prediction based on two dimensional prediction algorithms
}

\author{
Lei Xiao, Xue Yang, Min Zhu, Lipeng Zhu \\ ( State Grid Meishan Electric Power Supply Company, sichuan 620010, China);
}

Keyword: network flow; horizontal prediction; vertical prediction; two dimensional flow prediction

\begin{abstract}
There is obvious periodic data flow curves of the power integrated service network based on the full use of historical data, this paper makes full use of the historical data, the horizontal and vertical dimensions are extracted from historical data. In this paper, a two dimensional flow forecasting method is proposed by studying the existing network traffic prediction model. In this method, a single exponential smoothing algorithm is used to calculate the horizontal and vertical predictions. it combines the advantages of the two dimensional prediction models, and can get better prediction accuracy than the existing prediction algorithms at the turning point.
\end{abstract}

\section{Introduction}

With the rapid development of the network information and the network service in the electric power communication network, network traffic behavior is increasingly complex, and network business needs (image, video conference service, etc.) are increasing. In the face of the diversity of business and the difference between the information management and the operation of power network, the demand of intelligent electric power communication network is constantly improved, which brings new challenges to the network management and optimization of the communication transmission network. Therefore, forecast the change trend of network data flow, rationally allocate the limited cyber source, and do optimization network expansion plan are of great concern to solve the network congestion and provide users better service quality. This paper introduces the traditional traffic prediction methods, and this paper proposes a two dimensional prediction method for network traffic classification based on a comprehensive analysis of the characteristics of network traffic. This method can solve the problem of one step delay in the prediction of periodic traffic flow[1].

\section{Research on Traffic Forecasting Model of Power Communication Network}

At present commonly used network flow model including Poisson process、Markov model、 heavy-tailed ON/OFF model、discrete wavelet model and so on.Poisson process model is first proposed based on the characteristics of telephone service flow,it is good simulating the early modeling network traffic,however, poisson model already can't satisfy the actual test to obtain the flow characteristic of modern communication network.Markov model is based on poisson process model of network traffic with queuing performance , and it is easy to deal with time series, but the complexity of the model resolution is larger, at the same time it can only for short-term forecasting network traffic.ON/OFF model can well explain the self-similar network traffic phenomenon, but too strict assumptions of the model for each data source, because it must be independent identically distributed, and with a constant output rate by using the model of network traffic and will produce large error.ARIM and FARIMA is suitable for processing stationary series, at the same time they can also handle the nonstationary sequence that are seasonal, cyclical and tendency.

In this article, through the comprehensive analysis of the characteristic of network traffic, it is based on a comprehensive power network data flow curve exists obvious periodicity, accoring to this phenomenon, we propose a two-dimensional flow prediction method, using the single exponential smoothing algorithm to a transverse and longitudinal prediction calculated precision index, a reasonable combination of the two dimension prediction model, the advantages of this 
method can get higher than the existing prediction algorithm in the turning point of the prediction precision[2-3].

\section{Traffic prediction of electric power communication network based on two - dimensional forecasting algorithm}

\section{Network traffic analysis}

The network traffic of the integrated service network of the smart grid has obvious daily periodicity. There are some turning points in the flow curve, such as crest, trough and local peak. These turning points are roughly the same every day. At the same time there is a daily flow of the unique trend of the day, at some time it is clearly different from historical data. The two-dimensional prediction algorithm proposed in this paper can better capture the trend of data traffic on the same day. The vertical prediction can well reflect the position of the turning point in the integrated service network. Considering these two characteristics, The problem of prediction delay exists at the turning point of the previous algorithm is solved and the prediction accuracy of the trend section is guaranteed.

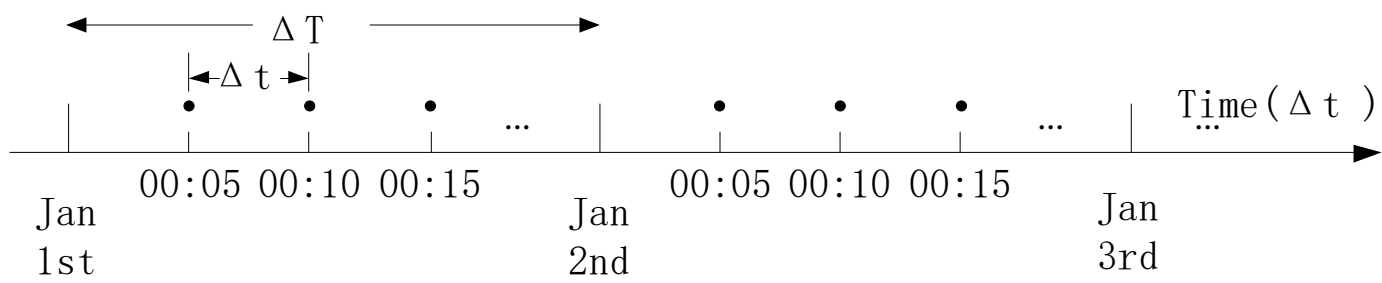

Figure.2.1. Horizontal dimension

As shown in Fig 2.1, the flow data at different times of the same period in the same date are horizontal dimension, such as the transverse flow data with sampling interval , is the sampling interval length, $\Delta \mathrm{T}$ is the day Cycle length. $\mathrm{S}(\mathrm{t}, \mathrm{d})$ is the sampled value at the No.t sampling time on day $\mathrm{d}$, where , , $m$ is the sum days of the historical data, so that for the horizontal data sequence, said as the length of the horizontal training data sequence, there are

$$
L x=n \frac{\Delta T}{\Delta t}(n \leq m)
$$

The data flow at the same time on different dates is the vertical dimension.

Let $y_{t}=\left\{y_{(t, i)} \mid i=1,2, \cdots, L y\right\}$ be longitudinal time series, where $L y$ is the length of the longitudinal sequence, there is

$$
y_{t}=\{S(t, d) \mid d=1,2, \cdots, L y ; L y \leq m\}
$$

\section{Network prediction model \\ Horizontal prediction}

Since the horizontal dimension has obvious daily periodicity, the random fluctuation component is less, and it is suitable for using the nonlinear prediction algorithm. Wavelet neural network algorithm with wavelet basis function instead of the previous S-type function as the hidden layer node transfer function, combined with multi-resolution wavelet analysis and neural network self-learning ability, this paper selects wavelet neural network algorithm as the horizontal line prediction algorithm, The Morlet mother wavelet function is chosen as the wavelet neural network implicit layer neuron equation, 2-5 is the expression, the function has higher time domain and frequency domain resolution[4-5].

$$
\varphi(t)=\cos (1.75 t) e^{-t^{2} / 2}
$$

The lateral prediction is to train the wavelet neural network by the transverse training sequence $x=\left\{x_{i} \mid i=1,2, \cdots, L x\right\}$. First, the data is processed and the training sequence is mapped to the 
interval $[-1,1]$ by the formula (2-6).

$$
\begin{aligned}
\overline{x_{i}}= & \left(v_{\max }-v_{\min }\right) *\left(x_{i}-x_{\min }\right) /\left(x_{\max }-x_{\min }\right) \\
& +v_{\min }(i=1,2, \ldots, m)
\end{aligned}
$$

Where $v_{\max }=1, v_{\min }=-1, x_{\max }=\max \left(x_{i}\right), x_{\min }=\min \left(x_{i}\right)$ 。Then, the number of nodes in each layer of wavelet neural network is determined and the number of nodes in the input layer is $N$, the number of nodes in the hidden layer is $H$ and the number of nodes in the output layer is $M, H$ is expressed as:

$$
H<\sqrt{N+M}+a \quad, a \in[0,10]
$$

The weights of the wavelet neural network and the coefficients of the wavelet basis function are constantly modified according to the prediction error until the training number reaches the preset number of training times or the prediction error reaches the preset error value. Using the trained model to predict, for the current time $t$, given wavelet neural network input layer data $\left\{x_{i} \mid i=t, t-1, \ldots, t-M+1\right\}$ to obtain the predicted output ${ }^{X_{t+1}}$.

\section{Vertical prediction}

Vertical dimension random fluctuation component is large, long-term trend is single, suitable for using simple linear prediction algorithm.In this paper, the ARIMA algorithm is selected as the longitudinal prediction algorithm. The predicted value of the ARIMA algorithm is self-latency variable and random variables linear combinations.And it suitable for non-stationary sequences,the algorithm is applied to non-stationary sequences made d-order difference to obtained and then predicted the stationary sequence.The mathematical expression of the model is:

$$
\Delta y_{t}=\varphi_{1} \Delta y_{t-1}+\varphi_{2} \Delta y_{t-2}+\ldots+\varphi_{p} \Delta y_{t-p}+u_{t}-\theta_{1} u_{t-1}-\theta_{2} u_{t-2}-\ldots-\theta_{q} u_{t-q}
$$

Among them $u_{i}(i=t, t-1, \ldots, t-p)$ is random item,$\varphi_{i}(i=1,2, \ldots q)$ is autoregressive coefficient, $\theta_{i}(i=1,2, \ldots q)$ is moving average coefficient, $\Delta y_{i}$ is $y_{i}$ sequence after d-order difference. $y_{i}$ sequence able to get after $\Delta y_{i}$ sequence made d cumulative.

The longitudinal prediction first tests whether the longitudinal data sequence is a stationary time series. If it is not to carry on the d-order difference to get the smooth sequence again.Then make sure $\operatorname{ARIMI}(p, d, q)$ model order $p$ and q.In this paper, the common AIC criterion is used to determine the model order.Select AIC $(p, q)$ smallest set of $p$, q is the order of the model,the formula is:

$$
\operatorname{AIC}(p, q)=N \ln \left(\sigma_{\varepsilon}^{2}\right)+2(p+q+1)
$$

Where $\mathrm{N}$ is the length of the sequence, the residual of the model. Finally, the unknown coefficients are estimated, and parameters of each sequence are estimated to determine the autoregressive coefficients and the moving average coefficients. The predicted model is used to predict the time series. At the time $t$ and $t$, the longitudinal sequence is input to the model, and its difference is obtained d times, and the predicted value is calculated[6].

\section{Two - dimensional prediction algorithm}

As shown in Figure 2-2, the horizontal and vertical dimensions of historical data are acquired, and the ARIMA and wavelet neural network algorithms are trained with the horizontal and vertical dimension data respectively. Then, the traffic data at time $t$ is read and the horizontal prediction Algorithm and longitudinal predictor are used to calculate the prediction values of the horizontal and vertical dimensions at time $\mathrm{t}+\mathrm{T}$ respectively. The final prediction output is obtained by summation of weights $\mathrm{w}$, and finally the weight $\mathrm{w}$ is updated by the single exponential smoothing algorithm return. 


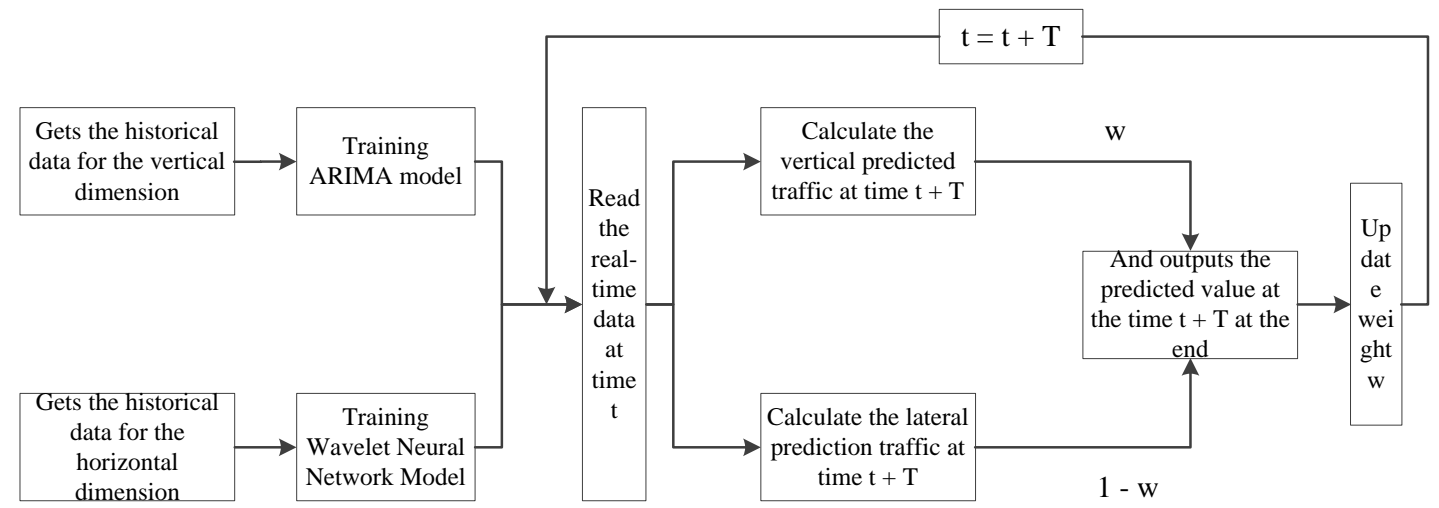

Figure 2-2 Two-dimensional prediction model

The mathematical expression of the single exponential smoothing algorithm is:

$S_{t}=\alpha X_{t}+(1-\alpha) S_{t-1}=\sum_{i=0}^{t} \alpha(1-\alpha)^{t-i} X_{i}$

Where $X_{t}$ is the value of the time series at time, is the predicted value of the time series at time $t+1$, and is the weight coefficient. For large fluctuating time series, the value is larger to increase the effect of the most recent data on the predicted value. For the relatively stable time series, the value is smaller to reduce the influence of the most recent data on the prediction results.

Define the prediction errors of the horizontal and vertical prediction at the time, respectively $e_{x t}, e_{y t}$,the final prediction error is

$e_{x t}=x_{t}^{\prime}-v_{t}$

$e_{y t}=y_{t}^{\prime}-v_{t}$

$e_{p t}=p_{t}-v_{t}$

In formula $V_{t}$ is the true value of the network traffic at the moment, $X_{t}$ is the lateral prediction predicts the value of the horizontal dimension at time, ${ }^{y^{\prime}{ }_{t}}$ is the longitudinal prediction predicts the predicted value at the moment in the longitudinal dimension, $\left\{w_{t}^{\prime} \mid w_{t}^{\prime} \in[0,1]\right\}$ is used (2-10) the weight coefficient of the predicted time. ${ }^{p_{t}}$ is the final predicted output.The expression is: $p_{t}=w_{t}^{\prime} x_{t}^{\prime}+\left(1-w_{t}^{\prime}\right) y_{t}^{\prime}$

To ensure that the performance prediction error $e_{p t}$ of the two-dimensional prediction algorithm must meet the following expression:

$\left|e_{p t}\right| \leq \min \left(\left|e_{x t}\right|,\left|e_{y t}\right|\right)$

Under the premise of the known $v_{t}$,find optimal value of $w_{t}$ made $\left|e_{p t}\right|$ min, the expression as follow:

$$
\begin{aligned}
\left|e_{p t}\right| & =\left|w_{t} x_{t}^{\prime}+\left(1-w_{t}\right) y_{t}^{\prime}-v_{t}\right| \\
& =\left|w_{t} e_{x t}+\left(1-w_{t}\right) e_{y t}\right|
\end{aligned}
$$

Obviously,when $e_{x t}$ and ${ }^{e_{y t}}$ same number, $e_{p t}= \pm\left(w_{t} e_{x t}+\left(1-w_{t}\right) e_{y t}\right), w_{t} \in[0,1], w_{t}=0$ and $w_{t}=1$ made min,and satisfy the formula (2-15). when $e_{x t}$ and $e_{y t}$ different number, ${ }^{\mid} e_{p t} \mid$ min is 0,at this time: 


$$
w_{t}=\left\{\begin{array}{cc}
\frac{e_{y t}}{e_{y t}-e_{x t}} & e_{x t} e_{y t} \neq 0 \\
0 & e_{x t} e_{y t}=0
\end{array}\right.
$$

Using $\left\{w_{i} \mid i=1,2, \ldots t\right\}$ to complete the prediction $w_{t+1}^{\prime}$ from the sequence, the completion of $w$ the dynamic weight update:

$$
w_{t+1}^{\prime}=\sum_{i=0}^{t} \alpha(1-\alpha)^{t-i} w_{i}
$$

In the formula, the minimum $\alpha$ MSE value is selected as the coefficient.

\section{Simulation and performance comparison}

In order to validate our proposed prediction analysis method,we collected from a certain electric power company core router traffic data of 218 days,including data sampling interval for 5 min/times,a total of 288 traffic data points a day.Selection in this paper,the simulation analysis of the continuous flow data of 22 days and 21 days will be divided into 22 days traffic data of the historical data and test data, 1 day transverse data training data sampling interval for 5 min,length of time for four days.Longitudinal training data using the historical data of 21 days,according to the time is divided into 288 groups, and the same time different date for a group,each group leader degrees for the 21st.

when 14 to 17 section lateral prediction turning point in the curve in a relatively real and estimated values lag phenomenon,and the longitudinal prediction in this section is relatively accurate,figure 3-2 shows in this interval weights of less than 0.5.At 17 to 19 interval,when the longitudinal prediction accuracy is decreased obviously,and lateral prediction is captured very well on the day of the trend,combined with figure 3 is shown in figure 3-2-1 and the simulation results confirmed the weights of the dynamic update strategy well complementary advantages of two dimensions.
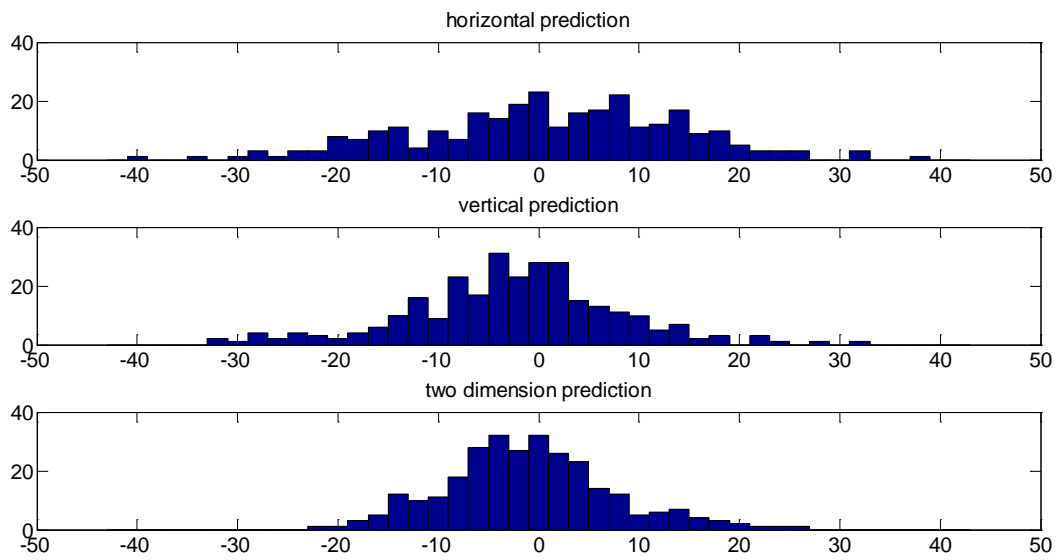

Figure 3-1 Prediction error distribution

Figure 3-1 for the transverse,longitudinal prediction and two-dimensional prediction error distribution,it can be seen that the two-dimensional prediction of small error range forecast precision is high,when the horizontal and vertical dimensions of predicted value is greater than or less than true value, the weight $\mathrm{w}$ values make small prediction error of the dimensions of 1.A one hour in horizontal and vertical prediction $\mathrm{w}$ value can make a general prediction error is less than any a dimension and approach to zero. 


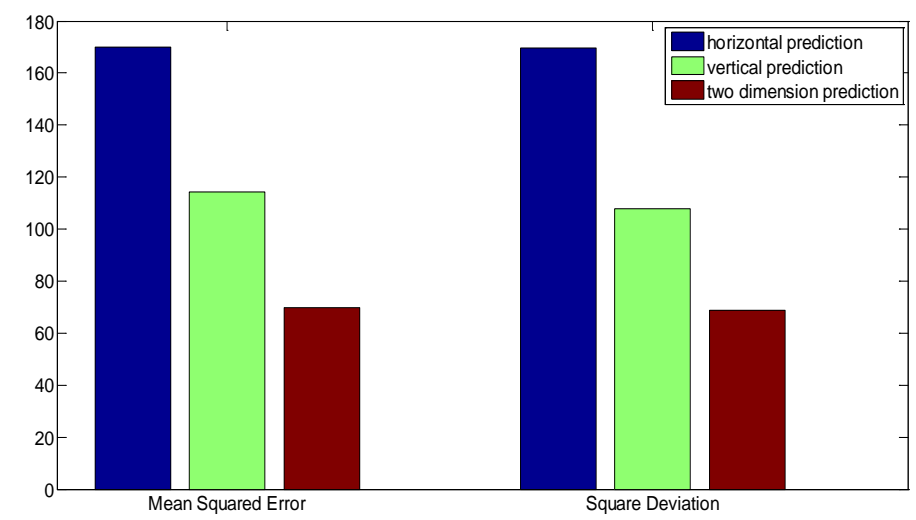

Figure 3-2 Predict MSE and variance comparison chart

Figure 3-2 MSE and variance comparison show that this paper proposed a two-dimensional prediction algorithm in prediction effect is better than two dimensions separately predict,at the same time, because this article update weights using single exponential smoothing algorithm, error fluctuation is less than a single dimension.Comprehensive the above analysis may safely draw the conclusion, this paper proposed a two-dimensional prediction algorithm by combining the advantage of two dimensions, effectively solved the problem of the turning point in the delay, at the same time improve the prediction accuracy of the algorithm.

\section{Conclusion}

Based on the study of the existing traffic prediction model of power communication network, this paper proposes a new method of power communication network traffic prediction based on two dimensional prediction algorithms. By making full use of historical data, the historical data can be extracted from the two dimensions of horizontal and vertical. The lateral dimension reflects the rising and falling trend of the forecast day, and ensures the prediction accuracy of the two dimensional prediction algorithms at the non- turning point. The vertical dimension reflects the position of the turning point of the day, and the daily periodicity of the flow is used to improve the prediction accuracy of the two dimensional prediction algorithms at the turning point. Two dimensional flow prediction method calculate the accuracy of lateral prediction and vertical prediction by using single exponential smoothing algorithm. The two dimensional flow prediction method combines the advantages of the two dimensional prediction model, and improves the prediction accuracy of the turning point while ensures the prediction accuracy of the non- turning point.

\section{Reference}

[1] A. Keshavarz-Haddad and R. H. Riedi, “Bounds on the benefit of network coding for wireless multicast and unicast,” IEEE Transactions on MobileComputing, vol. 13, pp. 102-115, 2014.

[2] T.-h. Kim, H. Choi, and H.-S. Park, “Centrality-based network coding node selection mechanism for improving network throughput," in16th International Conference on Advanced Communication Technology (ICACT), 2014, pp. 864-867.

[3] X. Fang, S. Misra, G. Xue, D. Yang, “Smart grid the new and improvedpower grid,” in IEEE Communications Surveys and Tutorials, vol.14,pp.944-980, December 2012.

[4] V. C. Gungor, D. Sahin, T. Kocak, S. Ergut, C. Buccella, C. Cecati, etal, "Smart grid technologies: communication technologies and standards,"IEEE transactions on Industrial informatics, vol.7, pp.529-539, September2011.

[5] J. Wang, "A process level network traffic prediction algorithm based on ARIMA model in smart substation,” in IEEE International Conference on Signal Processing, Communication and 
Computing (ICSPCC), pp.1-5, August 2013.

[6] W. Jin, X. Yong jun, "Prediction of smart substations' network traffic based on improved particle swarm wavelet neural networks,” IEEE International Symposium on, pp.1-7, May 2013. 